\title{
Application of VEE Pro Software for Measurement of MOS Device Parameters using C-V curve
}

\author{
Viranjay M. Srivastava, G.Singh \\ Electronics and Communication Engineering, \\ Jaypee University of Information Technology, \\ Solan, Himachal Pradesh-173215, India
}

\author{
K.S.Yadav \\ VLSI Design Group, Central Electronics Engineering \\ Research Institute (CEERI), \\ Pilani, Rajasthan-333031, India
}

\begin{abstract}
For a capacitor formed of MOS device using Metal-silicon dioxide-silicon (MOS) layers with an oxide thickness of $528 \AA$ (measured optically), some of the material parameters were found from the curve drawn between Capacitance vs Voltage $(\mathrm{C}-\mathrm{V})$ through the Visual Engineering Environment Programming (VEE Pro) software. To perform the measurment, process by a distance, from the hazardous room, we use VEE Pro software. In this research, to find good result, vary the voltage with smaller increments and perform the measurements by vary the applying voltage from $+7 \mathrm{~V}$ to $-7 \mathrm{~V}$ and then back to $+7 \mathrm{~V}$ again and then save this result in a Data sheet with respect to temperature, volage and frequency using this program.
\end{abstract}

\section{Keywords}

Capacitance-voltage curves; LCR Meter; MOS device; VEE Pro; VLSI;

\section{INTRODUCTION}

Wherever VEE is a graphical programming environment optimized for use with electronic instruments. The simplest way to state its value is the phrase, "Better measurements faster". Software prototyping is noticeably faster with VEE than with lower-level languages, and hundreds of powerful functions are available to help for analyze the measurement data.

The VEE programming environment strongly supports electronic instrument and measurement tasks. For those early prototyping stages, VEE provides simulated signal sources and displays. We can experiment with program flow and data processing without any external hardware [1].

When we are ready for real-world data, VEE provides an instrument manager and a dynamic I/O server to simplify the tasks of discovering, configuring, and managing external instruments. VEE supports several types of instrument drivers and lets you choose the driver that best suits our preferences and/or the characteristics of an instrument. For those times when the instruments are not available, just press one button in the Instrument Manager to take a driver "off line" and continue to developing your program.

By this research we can easily extract the oxide thickness, flat band capacitance, flat band voltage, threshold voltage, metalsemiconductor work function.

\subsection{Selecting E4980A Precision L-C-R meter}

Designed for measurement precision and ease of use, this family of LCR meters fits both R\&D and production applications. Although the LCR meter does not have all the sophisticated features as impedance analyzers, the LCR meter shown in Fig.1, offers excellent performance at an affordable cost.The properties of LCR meter can be given as following,

1. Wide selection of frequency range from $20 \mathrm{~Hz}$ to $3 \mathrm{GHz}$.

2. Frequency list sweep for continuous testing at multiple frequency points.

3. Used for general purpose testing of surface-mount components, leaded components, materials and more.

4. GPIB and handler interface for easy test automation in production environment.

5. It has $0.05 \%$ basic accuracy

6. Measurement parameters: $|\mathrm{Z}|,|\mathrm{Y}|, \theta, \mathrm{R}, \mathrm{X}, \mathrm{G}, \mathrm{B}, \mathrm{L}, \mathrm{C}, \mathrm{D}, \mathrm{Q}$ Rdc, Idc, Vdc.

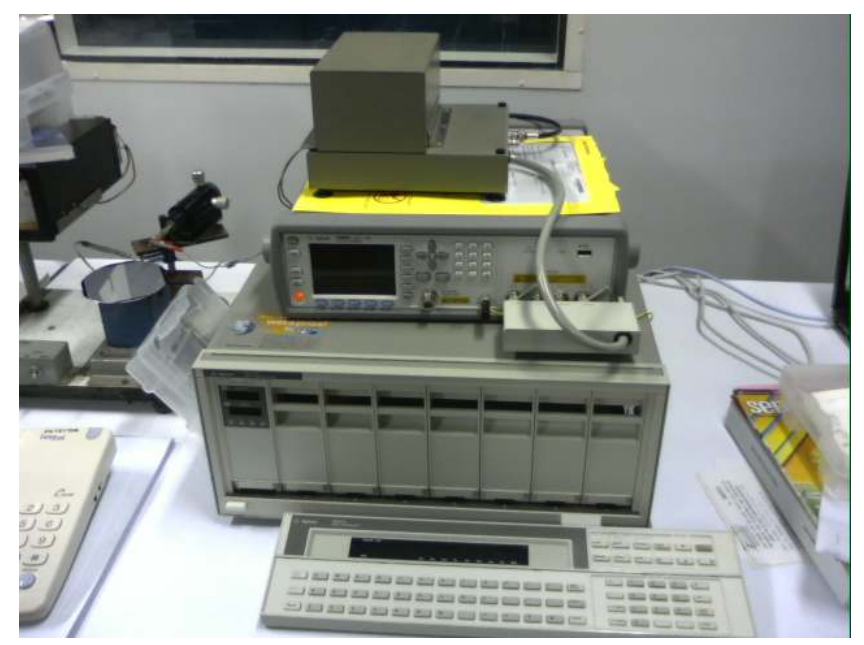

Figure 1. LCR meter 


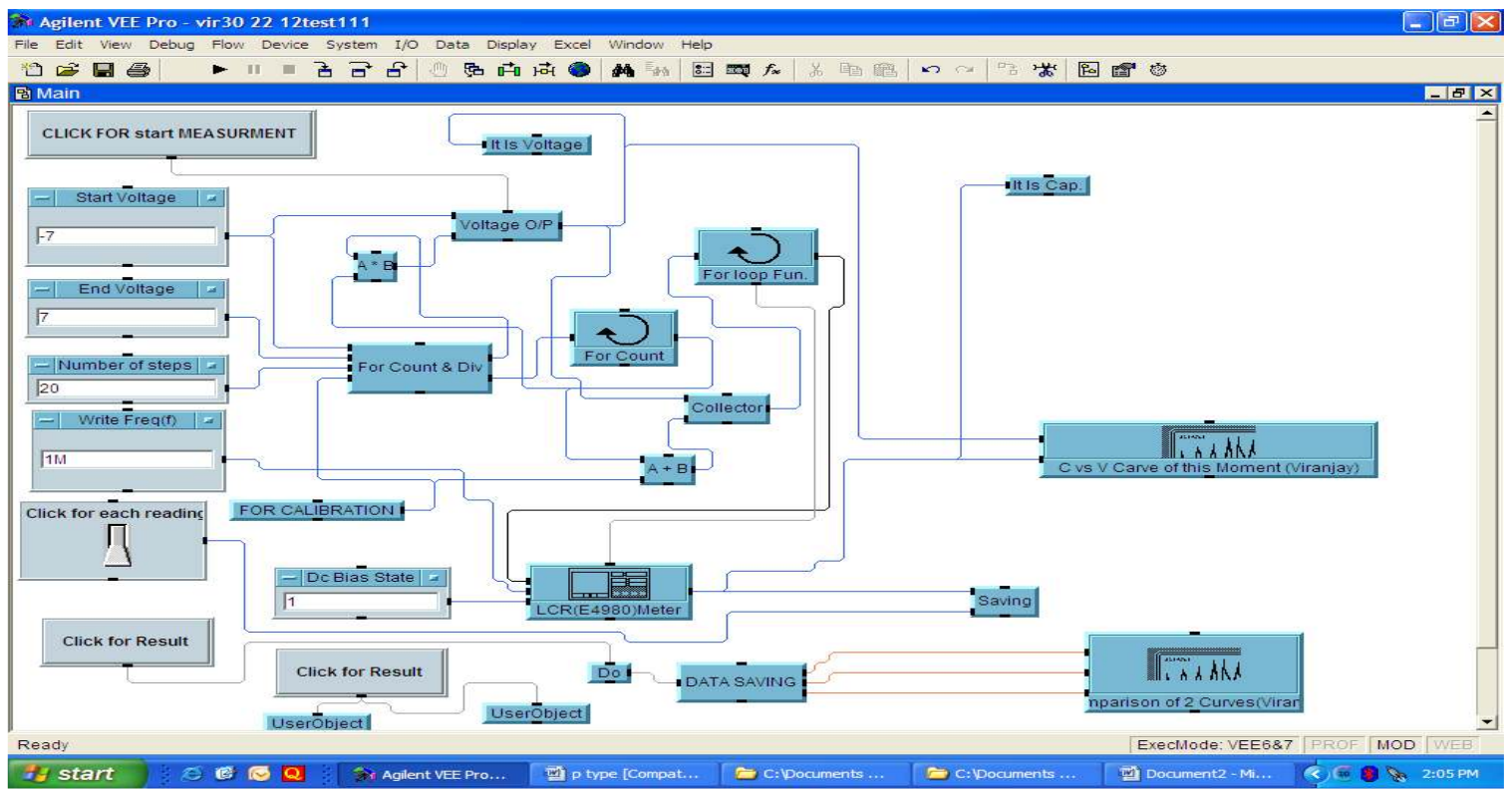

Figure 2. Main programme (Block diagram)

\section{EXPERIMENTS}

To perform this experiment, for measurment of capacitances at different voltages [2], mainly for a voltage range, author will make a programme using the programming tools in VEE Pro software which is interface with the LCR meter as shown in Fig. 1 step by step programming is given in following stages [3, 4].

\subsection{To set the range for voltage}

Since for the measurment, first, select the Input range of voltages for which the capacitance measurments are required [5]. Procedure of this programming is shown in Fig. 2. For this purpose the programming is done on basis that to set the parts, take the difference of maximum and minimum voltage (for voltage range), and divide them by the number of steps, at which capacitances are to be measured. After that addition, this small division to the minimum voltage as in Fig. 2, one by one, untill we reach to maximum voltage, this is done in my programming using loop bearing name "for count and division" as shown in Fig. 3 and Here a switch is added (bearing block named "click for each reading"), by which two readings can be taken, one before heating and other after heating, so that better variation of capacitances and performance can be observed.

For example in this research we take the range between $-7 \mathrm{v}$ to $+7 \mathrm{v}$, and it is divided into 20 parts, so that one division comes of $0.7 \mathrm{v}$, we will add this to $-7 \mathrm{v}$ and after 20 steps it will reach to it's maximum value of $+7 \mathrm{v}$.

\subsection{To set the frequency}

In this part of programming, set the frequency at which reading of capacitances are required (at low frequency or quasistatic and at high frequency) [6]. This frequency can vary from $10 \mathrm{~Hz}$ to 1 $\mathrm{MHz}$. This frequencies will required for different types of MOS (N-type or P-type). For this purpose we have to write only the value of frequency, not the unit in the block of required frequency. As in Fig. 2, the reading is taken at $1 \mathrm{MHz}$ and it is written as $1 \mathrm{M}$.

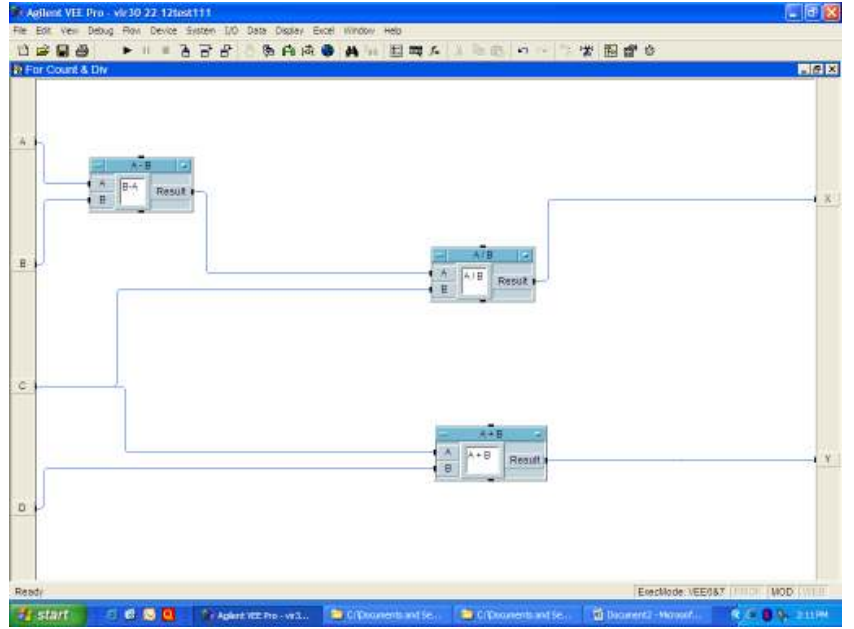

Figure 3. Count and division block

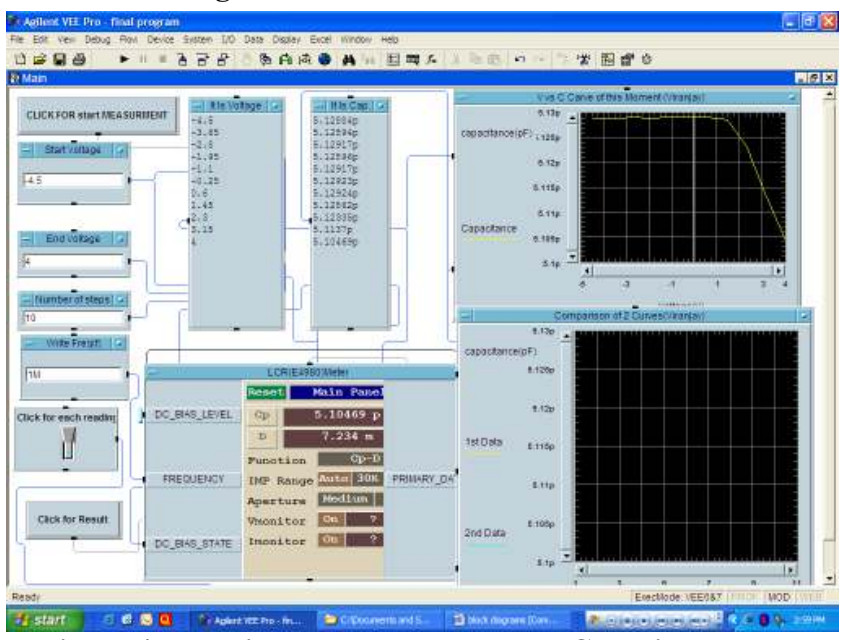

Figure 4. Readings and graph between Capacitances and voltages (Before heating) 


\subsection{To draw curve on graph sheet of system}

After setting the volage range and frequency, we can take the reading on window of program, also the graph of capacitances at these voltages are drawn by using this program on graphs) respectively. The arrangment for heating system, MOS device and probe through which device and LCR meters are connected, are shown in Fig. 1. The purpose of heating the device is to get accurate measurment of parameters due to the effect of charges in oxide layer. For p-type MOS, graph is shown in Fig. 4.

\subsection{To save the result}

After getting data on graph sheet of programming window, It is required to save this on an excel sheet as described in Fig. 6, where calculation for measurment of parameters as oxide thickness, flat band capacitance and flat band voltage, threshold voltage, metal-semiconductor work function and differences in capacitances before and after the heating the device can be calculated directly using standard formulas.

\subsection{To compare the capacitances}

When we get the two different curves of capacitance vs voltage, one before heating and other after heating (yellow coloured/solid line shows before heating curve and green coloured/dashed line shows after heating curve), the value of the capacitances can be compared directly by curve or by data on excel sheet. If curves or data are within the limit as shown in Fig. 8 and Fig. 9, then MOS device is good for different applications, but if these readings are out of limit then device will not be used for further applications and fabrication process will be checked for better performance production of MOS devices. These limits can be given by the costumer, who will use these devices for different applications. By this program, from a remote place, we can measure the correctness of MOS devices which are fabricated in hazardous lab or chamber.

\section{RESULT}

Using the VEE Pro software with above programming to stores data on Excel sheet, parameters as oxide thickness, flat band capacitance and flat band voltage, threshold voltage and metalsemiconductor work function difference can be automatically calculated from test data at the end of the $\mathrm{C}-\mathrm{V}$ test, and then this calculated parameters can be automatically entered back into the data worksheet for record purpose.

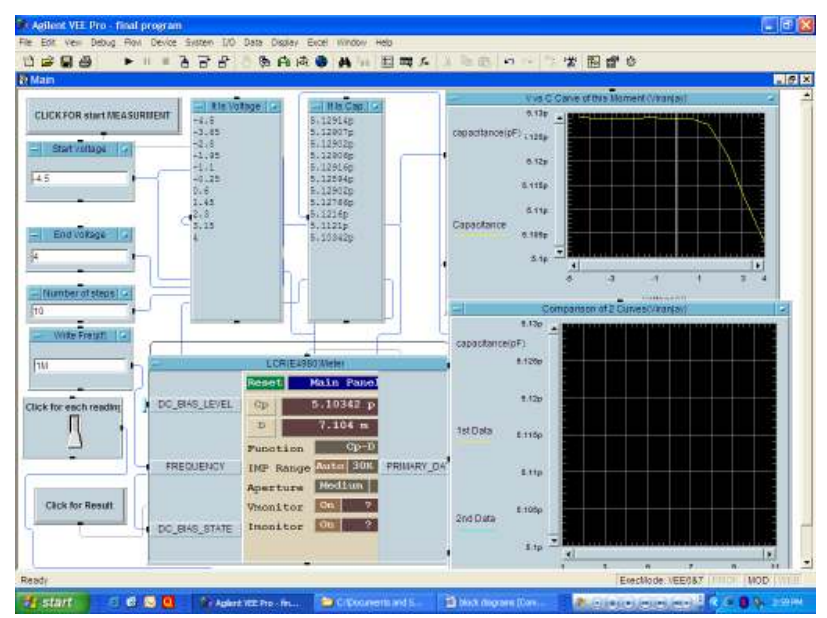

Figure 5. Readings and graph between Capacitances and voltages (After heating)

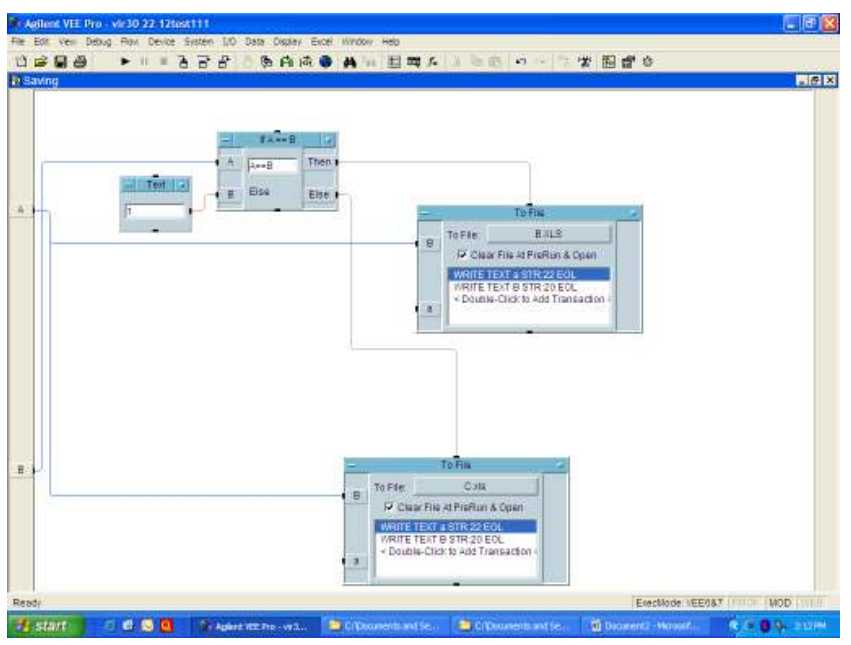

Figure 6. Saving data of capacitance, voltage, frequency

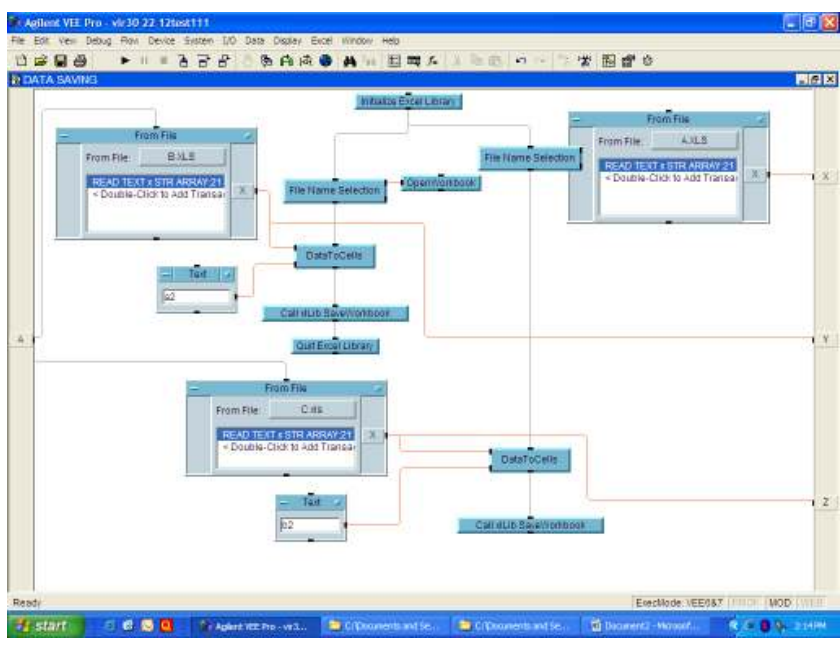

Figure 7. Saving data on Excel sheet

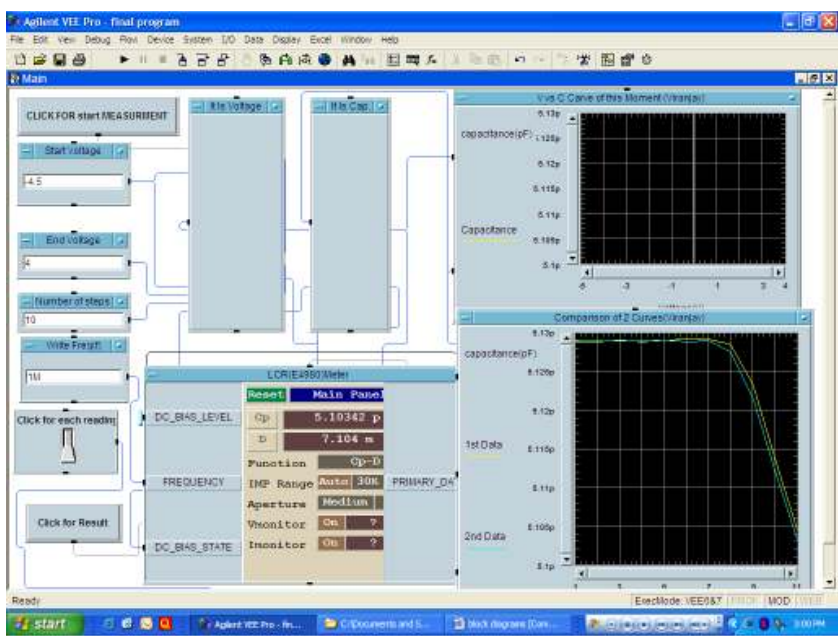

Figure 8. Comparison of curves before and after heating 


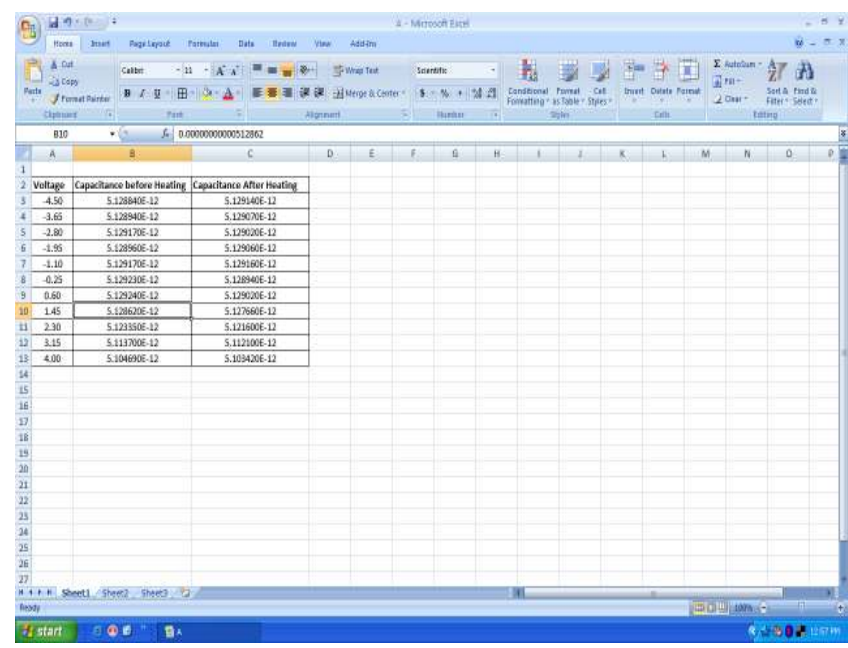

Figure 9. Comparison of data of capacitances before heating and after heating on Excel sheet

\section{CONCLUSION}

The real importance of Capacitance-Voltage curve measurement techniques is that a large number of device parameters can be extracted from two seemingly simple curves: the high frequency $\mathrm{C}-\mathrm{V}$ curve and the quasistatic $\mathrm{C}-\mathrm{V}$ curve. These parameters can provide critical device and process information.

Based on the C-V characterization of a MOS capacitor with VEE Pro software in lab of BEL, which resulted in erroneous values of device parameters, mainly the substrate dopant concentration, on which most of the other parameters are based [9, 10]. The readings stored in excel sheet can be used for further processing.

In this experiment we can also identified the improper contact of the probes with the wafer surface which is connected to LCR meter as the most probable cause of that along with the possibility of going unintentionally into deep depletion or having the equipment improperly calibrated. This can give erroneous result.

\section{ACKNOWLEDGMENTS}

The authors would like to thank Ms. Rejeena R.Y., Fabrication Laboratory, BEL, Bangalore, India where the devices were fabricated. The authors want to thank Prof. A.B. Bhattachharaya, Jaypee University, Noida, India and Mr. Peeyush Tripathi, Intel, Bangalore, India for many insightful discussions.

\section{REFERENCES}

[1] “Agilent VEE pro user's Guide," Agilent Company.

[2] Thamos J. Mego, "Guidelines for Interpreting CV data," Solid State Technology, p.p. 159-163, May’1990.

[3] Popa, Mircea Ionel, Raul, "Virtual Instrumentation Application for System Identification," Instrumentation and Measurement Technology Conference, Proceedings of the IEEE, p.p. 842 - 846, April 2006.

[4] “HP VEE Reference," Hewlett Packard Company, $4^{\text {th }}$ edition, January 1995.

[5] “MOS Application note-1," MSI electronics, Woodside, N.Y. - 1137 (Manual)

[6] Thamos J. Mego, "On line C-V doping profile measurement of low dose ion implant", IEEE transaction on electron devices, vol. 27, no. 12, December 1980, 2268-2273

[7] Viranjay M. Srivastava, "Relevance of VEE Programming for Measurement of MOS Device Parameters," IEEE International Advance Computing Conference, India, 2009, p.p. 205-209

[8] Viranjay M. Srivastava, "Capacitance-Voltage Measurement for Characterization of a Metal-Gate MOS Process", International Journal of Recent Trends in Engineering (IJRTE), vol. 1, no. 4, May 2009, India, 4-7. http://www.academypublisher.com/ijrte/vol01/no04/ijrte0104 004007.pdf

[9] Nicollian, E.H. and Brews, J.R., "MOS Physics and Technology," Wiley, New York, 1982

[10] Sze, S.M., "Physics of Semiconductor Devices," $2^{\text {nd }}$ edition, Wiley, New York, 1985 